\title{
Elliptic flow coefficients from transverse momentum conservation
}

\author{
Adam Bzdak ${ }^{1, *}$ and Guo-Liang $\mathrm{Ma}^{2, \dagger}$ \\ ${ }^{1}$ AGH University of Science and Technology, Faculty of Physics and Applied Computer Science, 30-059 Kraków, Poland \\ ${ }^{2}$ Shanghai Institute of Applied Physics, Chinese Academy of Sciences, Shanghai 201800, China
}

(Received 27 October 2017; published 17 January 2018)

\begin{abstract}
We calculate the $k$-particle $(k=2,4,6,8)$ azimuthal cumulants resulting from the conservation of transverse momentum. We find that $c_{2}\{k\}>0$ and, depending on the transverse momenta, $c_{2}\{k\}$ can reach substantial values even for a relatively large number of particles. The impact of our results on the understanding of the onset of collectivity in small systems is emphasized.
\end{abstract}

DOI: 10.1103/PhysRevC.97.014903

\section{INTRODUCTION}

Relativistic heavy-ion collisions can create an extreme experimental environment with high temperature or high baryon chemical potential that possibly liberates quarks and gluons into a deconfined partonic matter, namely a quarkgluon plasma (QGP) [1,2]. The Relativistic Heavy-Ion Collider (RHIC) and the Large Hadron Collider (LHC) have been devoted to investigating the properties of QGP and its transition to hadronic matter. Many experimental results indicate that a strongly interacting partonic matter has been produced in high-energy nucleus-nucleus $(A+A)$ collisions at both RHIC and the LHC [3-5]. One of the most important experimental observations is the collective flow phenomenon, which is believed to arise from the hydrodynamical evolution of QGP, which can convert the initial geometry fluctuations into the final particle momentum anisotropies [6-8]. Different experimental methods were invented to measure the coefficients of collective flow $v_{n}$, e.g., the event plane $v_{n}\{E P\}$ method, two-particle correlations $v_{n}\{2\}$, or multi-particle cumulants $v_{n}\{k\}$ [9-12], which show different sensitivities to flow and nonflow effects $[13,14]$. Hydrodynamical models have successfully reproduced the main features of the measured flow coefficients, see, e.g., Refs. [15-20]. It indicates that a strongly interactive and collective matter has been produced in $A+A$ collisions.

Recent experimental results showed long range in rapidity (i.e., across a large rapidity gap) azimuthal correlations in high-multiplicity proton-proton $(p+p)$ and proton-nucleus $(p+A)$ collisions. The extracted $v_{n}$ coefficients are comparable to those measured in $A+A$ collisions [21-24], see also a

\footnotetext{
*bzdak@fis.agh.edu.pl

†'glma@sinap.ac.cn
}

Published by the American Physical Society under the terms of the Creative Commons Attribution 4.0 International license. Further distribution of this work must maintain attribution to the author(s) and the published article's title, journal citation, and DOI. Funded by $S C O A P^{3}$. recent experimental review [25]. Similar effects were observed in $p+\mathrm{Au}, d+\mathrm{Au}$, and ${ }^{3} \mathrm{He}+\mathrm{Au}$ collisions at RHIC [26-28], indicating the importance of initial geometry on the observed azimuthal anisotropies. As already discussed in Refs. [29,30] these results can be described by hydrodynamical and transport models, see, e.g., Refs. [8,31-41], indicating that collective flow also exists in small but dense systems. The more detailed experimental measurements find that the elliptic flow coefficient is smaller in $p+p$ and $p+\mathrm{Pb}$ than in $\mathrm{Pb}+\mathrm{Pb}$ for a given multiplicity at the LHC energy, but it does not seem to turn off at low multiplicities [42,43]. ${ }^{1}$ These features seem to challenge our current understanding of collectivity based on the hydrodynamical picture, since it is expected that hydrodynamics should not be applicable to low-multiplicity events, where the initial state effects are expected to be well visible, see, e.g., Refs. [45-51]. See also Refs. [52-55] for other related ideas.

To shed more light on the low multiplicity collectivity we will explore the effect of transverse momentum conservation (TMC), which is a well-known azimuthal correlation between all produced particles [56-61]. TMC is an important background in direct flow $v_{1}$ measurements, where its influence has to be corrected especially in peripheral $A+A$ collisions $[12,62]$, where $1 / N$ corrections due to TMC can be sizable. Therefore, it is essential to explore how TMC influences the elliptic flow coefficients $v_{2}\{k\}$ in small systems, where the multiplicity could be very low and the effect of TMC could be significant. In particular, the presumed long-range character of TMC complicates the interpretation of the subevent cumulant method proposed recently in Refs. [63,64].

In this paper we calculate

$$
c_{2}\{2\}=\left\langle e^{i 2\left(\phi_{1}-\phi_{2}\right)}\right\rangle
$$

\footnotetext{
${ }^{1} v_{2}\{2\}$ can even increase with decreasing event multiplicity in $d+\mathrm{Au}$ collisions at RHIC energies [44], which is likely due to the nonflow effects, such as the conservation of transverse momentum discussed in this paper.
} 
originating from the conservation of transverse momentum. We also calculate the leading terms of higher-order correlation functions [9-12,43]

$$
\begin{gathered}
c_{2}\{4\}=\left\langle e^{i 2\left(\phi_{1}+\phi_{2}-\phi_{3}-\phi_{4}\right)}\right\rangle-2\left\langle e^{i 2\left(\phi_{1}-\phi_{2}\right)}\right\rangle^{2} \\
c_{2}\{6\}=\left\langle e^{i 2\left(\phi_{1}+\phi_{2}+\phi_{3}-\phi_{4}-\phi_{5}-\phi_{6}\right)}\right\rangle \\
-9\left\langle e^{i 2\left(\phi_{1}-\phi_{2}\right)}\right\rangle\left\langle e^{i 2\left(\phi_{1}+\phi_{2}-\phi_{3}-\phi_{4}\right)}\right\rangle+12\left\langle e^{i 2\left(\phi_{1}-\phi_{2}\right)}\right\rangle^{3},
\end{gathered}
$$

and

$$
\begin{aligned}
c_{2}\{8\}= & \left\langle e^{i 2\left(\phi_{1}+\phi_{2}+\phi_{3}+\phi_{4}-\phi_{5}-\phi_{6}-\phi_{7}-\phi_{8}\right)}\right\rangle \\
& -16\left\langle e^{i 2\left(\phi_{1}-\phi_{2}\right)}\right\rangle\left\langle e^{i 2\left(\phi_{1}+\phi_{2}+\phi_{3}-\phi_{4}-\phi_{5}-\phi_{6}\right)}\right\rangle \\
& -18\left\langle e^{i 2\left(\phi_{1}+\phi_{2}-\phi_{3}-\phi_{4}\right)}\right\rangle^{2} \\
& +144\left\langle e^{i 2\left(\phi_{1}-\phi_{2}\right)}\right\rangle^{2}\left\langle e^{i 2\left(\phi_{1}+\phi_{2}-\phi_{3}-\phi_{4}\right)}\right\rangle-144\left\langle e^{i 2\left(\phi_{1}-\phi_{2}\right)}\right\rangle^{4},
\end{aligned}
$$

which are directly measured at RHIC and the LHC. If $c_{2}\{k\}$ cumulants are free of nonflow effects, then they can be related to the $k$-particle elliptic flow coefficients, $v_{2}\{k\}$, using

$$
\begin{aligned}
& \left(v_{2}\{2\}\right)^{2}=c_{2}\{2\}, \quad\left(v_{2}\{4\}\right)^{4}=-c_{2}\{4\}, \quad\left(v_{2}\{6\}\right)^{6}=\frac{c_{2}\{6\}}{4}, \\
& \left(v_{2}\{8\}\right)^{8}=-\frac{c_{2}\{8\}}{33} .
\end{aligned}
$$

We find that TMC results in $c_{2}\{k\}>0$ for the calculated $k=2,4,6,8$. In addition, $c_{2}\{k\} \sim 1 / N^{k}$ for large $N$, where $N$ is the number of particles subjected to transverse momentum conservation.

In the next section we present the details of our calculations. Next we discuss the implications of our results and in the last section we give our conclusions.

\section{Calculation}

In our calculations we assume that transverse momentum conservation is the only source of correlations between final particles. By $\vec{p}_{i}$ we denote the transverse momentum of the $i$ th particle emitted in a collision. The $N$-particle transverse momentum distribution (normalized to unity) $f_{N}$ with imposed transverse momentum conservation is given by ${ }^{2}$

$$
f_{N}\left(\vec{p}_{1}, \ldots, \vec{p}_{N}\right)=\frac{1}{A} \delta^{2}\left(\vec{p}_{1}+\ldots+\vec{p}_{N}\right) f\left(\vec{p}_{1}\right) \cdots f\left(\vec{p}_{N}\right),
$$

where $f(\vec{p})$ is the single-particle transverse momentum distribution and

$$
A=\int_{F} \delta^{2}\left(\vec{p}_{1}+\ldots+\vec{p}_{N}\right) f\left(\vec{p}_{1}\right) \cdots f\left(\vec{p}_{N}\right) d^{2} \vec{p}_{1} \cdots d^{2} \vec{p}_{N},
$$

\footnotetext{
${ }^{2} N$ is the total (full phase space) number of particles when the global conservation of transverse momentum is considered. However, as pointed out in Ref. [60], the local (in rapidity) conservation of transverse momentum is likely more physical and thus $N$ can be smaller than the total number of particles.
}

with the integral taken over the full phase space denoted by $F$. The details of this calculation can be found in, e.g., Refs. [56,58,59,61].

After integrating out all but $k$ momenta we obtain

$$
\begin{aligned}
& f_{k}\left(\vec{p}_{1}, \ldots, \vec{p}_{k}\right)=\frac{1}{A} f\left(\vec{p}_{1}\right) \cdots f\left(\vec{p}_{k}\right) \\
& \times \int_{F} \delta^{2}\left(\vec{p}_{1}+\ldots+\vec{p}_{N}\right) f\left(\vec{p}_{k+1}\right) \cdots f\left(\vec{p}_{N}\right) d^{2} \vec{p}_{k+1} \cdots d^{2} \vec{p}_{N},
\end{aligned}
$$

which, using the central limit theorem, can be approximated $\mathrm{by}^{3}$

$$
\begin{aligned}
f_{k}\left(\vec{p}_{1}, \ldots, \vec{p}_{k}\right)= & f\left(\vec{p}_{1}\right) \cdots f\left(\vec{p}_{k}\right) \frac{N}{N-k} \\
& \times \exp \left[-\frac{\left(\vec{p}_{1}+\ldots+\vec{p}_{k}\right)^{2}}{(N-k)\left\langle p^{2}\right\rangle_{F}}\right],
\end{aligned}
$$

where

$$
\left\langle p^{2}\right\rangle_{F}=\frac{\int_{F} p^{2} f(\vec{p}) d^{2} \vec{p}}{\int_{F} f(\vec{p}) d^{2} \vec{p}}
$$

with the integration over the full phase space $F$.

\section{A. Two particles}

For two particles we obtain

$$
\begin{aligned}
f_{2}\left(\vec{p}_{1}, \vec{p}_{2}\right)= & f\left(\vec{p}_{1}\right) f\left(\vec{p}_{2}\right) \frac{N}{N-2} \\
& \times \exp \left[-\frac{p_{1}^{2}+p_{2}^{2}+2 p_{1} p_{2} \cos \left(\phi_{1}-\phi_{2}\right)}{(N-2)\left\langle p^{2}\right\rangle_{F}}\right],
\end{aligned}
$$

where $p_{i}=\left|\vec{p}_{i}\right|$ and $\phi_{1}-\phi_{2}$ is the azimuthal angle difference between the two particles.

Using

$$
\left\langle e^{i 2\left(\phi_{1}-\phi_{2}\right)}\right\rangle=\frac{\int_{0}^{2 \pi} f_{2}\left(\vec{p}_{1}, \vec{p}_{2}\right) e^{i 2\left(\phi_{1}-\phi_{2}\right)} d \phi_{1} d \phi_{2}}{\int_{0}^{2 \pi} f_{2}\left(\vec{p}_{1}, \vec{p}_{2}\right) d \phi_{1} d \phi_{2}},
$$

we obtain $c_{2}\{2\}$ at a given $p_{1}$ and $p_{2}$,

$$
\left.c_{2}\{2\}\right|_{p_{1}, p_{2}}=\frac{I_{2}(x)}{I_{0}(x)}, \quad x=\frac{2 p_{1} p_{2}}{(N-2)\left\langle p^{2}\right\rangle_{F}},
$$

where $I_{k}(x)$ is the modified Bessel function of the the first kind. ${ }^{4}$ Expanding Eq. (13) in powers of $x, I_{2}(x) / I_{0}(x) \approx \frac{x^{2}}{8}$, we obtain

$$
\left.c_{2}\{2\}\right|_{p_{1}, p_{2}} \approx \frac{p_{1}^{2} p_{2}^{2}}{2(N-2)^{2}\left\langle p^{2}\right\rangle_{F}^{2}}, \quad p_{1} p_{2}<\frac{1}{2}(N-2)\left\langle p^{2}\right\rangle_{F} .
$$

\footnotetext{
${ }^{3}$ See, e.g., Ref. [65] where the influence of TMC on the particle momentum spectra was calculated exactly, i.e., without using the central limit theorem.

${ }^{4}$ Additionally, we have $c_{1}\{2\}=-I_{1}(x) / I_{0}(x) \sim-1 / N$ and $c_{3}\{2\}=$ $-I_{3}(x) / I_{0}(x) \sim-1 / N^{3}$.
} 
Finally, let us calculate the integrated $c_{2}\{2\}$ over a given transverse momentum interval. It turns out that

$$
c_{2}\{2\}=\frac{\int d p_{1} d p_{2} p_{1} p_{2} f\left(p_{1}\right) f\left(p_{2}\right) \exp \left[-\frac{p_{1}^{2}+p_{2}^{2}}{(N-2)\left\langle p^{2}\right\rangle_{F}}\right] I_{2}\left[\frac{2 p_{1} p_{2}}{(N-2)\left\langle p^{2}\right\rangle_{F}}\right]}{\int d p_{1} d p_{2} p_{1} p_{2} f\left(p_{1}\right) f\left(p_{2}\right) \exp \left[-\frac{p_{1}^{2}+p_{2}^{2}}{(N-2)\left\langle p^{2}\right\rangle_{F}}\right] I_{0}\left[\frac{2 p_{1} p_{2}}{(N-2)\left\langle p^{2}\right\rangle_{F}}\right]},
$$

can be very well approximated by

$$
c_{2}\{2\} \simeq \frac{I_{2}(\hat{x})}{I_{0}(\hat{x})}, \quad \hat{x}=\frac{2 \sqrt{\left\langle p_{1}^{2}\right\rangle_{\Omega}\left\langle p_{2}^{2}\right\rangle_{\Omega}}}{(N-2)\left\langle p^{2}\right\rangle_{F}},
$$

where $\left\langle p^{2}\right\rangle_{\Omega}$ is the average of $p^{2}$ in a measured transverse momentum interval denoted by $\Omega$. For not-too-large momenta, $\hat{x}<1$, we obtain

$$
c_{2}\{2\} \approx \frac{\left\langle p_{1}^{2}\right\rangle_{\Omega}\left\langle p_{2}^{2}\right\rangle_{\Omega}}{2(N-2)^{2}\left\langle p^{2}\right\rangle_{F}^{2}} .
$$

\section{B. Four particles}

For four particles we have

$$
\begin{aligned}
f_{4}\left(\vec{p}_{1}, \ldots, \vec{p}_{4}\right)= & f\left(\vec{p}_{1}\right) \cdots f\left(\vec{p}_{4}\right) \frac{N}{N-4} \\
& \times \exp \left[-\frac{p_{1}^{2}+p_{2}^{2}+p_{3}^{2}+p_{4}^{2}}{(N-4)\left\langle p^{2}\right\rangle_{F}}\right] \exp (-\Phi),
\end{aligned}
$$

where

$$
\Phi=\frac{2}{(N-4)\left\langle p^{2}\right\rangle_{F}} \sum_{i, j=1 ; i<j}^{4} p_{i} p_{j} \cos \left(\phi_{i}-\phi_{j}\right)
$$

To calculate the four-particle correlator at a given transverse momenta $p_{1}, p_{2}, p_{3}$, and $p_{4}$,

$$
\begin{aligned}
& \left.\left\langle e^{i 2\left(\phi_{1}+\phi_{2}-\phi_{3}-\phi_{4}\right)}\right\rangle\right|_{p_{1}, p_{2}, p_{3}, p_{4}} \\
& =\frac{\int_{0}^{2 \pi} d \phi_{1} \cdots d \phi_{4} \exp (-\Phi) e^{i 2\left(\phi_{1}+\phi_{2}-\phi_{3}-\phi_{4}\right)}}{\int_{0}^{2 \pi} d \phi_{1} \cdots d \phi_{4} \exp (-\Phi)},
\end{aligned}
$$

we expand $\exp (-\Phi)$ in $\Phi$. In the numerator, denoted by $U$, the first nonvanishing term is given by $\Phi^{4} / 24$, which gives

$$
U \approx 24 \pi^{4} \frac{\left(p_{1} p_{2} p_{3} p_{4}\right)^{2}}{(N-4)^{4}\left\langle p^{2}\right\rangle_{F}^{4}},
$$

and the remaining terms are suppressed by the higher powers of $N$. In the denominator, denoted by $D$, we simply have $D \approx$ $16 \pi^{4}$ resulting in

$$
\left.\left\langle e^{i 2\left(\phi_{1}+\phi_{2}-\phi_{3}-\phi_{4}\right)}\right\rangle\right|_{p_{1}, p_{2}, p_{3}, p_{4}} \approx \frac{3}{2} \frac{\left(p_{1} p_{2} p_{3} p_{4}\right)^{2}}{(N-4)^{4}\left\langle p^{2}\right\rangle_{F}^{4}},
$$

and using Eq. (2) we find

$$
\left.c_{2}\{4\}\right|_{p_{1}, p_{2}, p_{3}, p_{4}} \approx \frac{\left(p_{1} p_{2} p_{3} p_{4}\right)^{2}}{(N-4)^{4}\left\langle p^{2}\right\rangle_{F}^{4}} .
$$

To obtain this result we replaced $N-2$ by $N-4$ in Eq. (14).

\section{Six and eight particles}

Performing analogous calculations we obtain ${ }^{5}$

$$
\left.\left\langle e^{i 2\left(\phi_{1}+\phi_{2}+\phi_{3}-\phi_{4}-\phi_{5}-\phi_{6}\right)}\right\rangle\right|_{p_{1}, \ldots, p_{6}} \approx \frac{45}{4} \frac{\left(p_{1} p_{2} p_{3} p_{4} p_{5} p_{6}\right)^{2}}{(N-6)^{6}\left\langle p^{2}\right\rangle_{F}^{6}}
$$

and

$$
\begin{gathered}
\left.\left\langle e^{i 2\left(\phi_{1}+\phi_{2}+\phi_{3}+\phi_{4}-\phi_{5}-\phi_{6}-\phi_{7}-\phi_{8}\right)}\right\rangle\right|_{p_{1}, \ldots, p_{8}} \\
\approx \frac{315}{2} \frac{\left(p_{1} p_{2} p_{3} p_{4} p_{5} p_{6} p_{7} p_{8}\right)^{2}}{(N-8)^{8}\left\langle p^{2}\right\rangle_{F}^{8}},
\end{gathered}
$$

resulting in

$$
\left.\frac{1}{4} c_{2}\{6\}\right|_{p_{1}, \ldots, p_{6}} \approx \frac{3}{2} \frac{\left(p_{1} p_{2} p_{3} p_{4} p_{5} p_{6}\right)^{2}}{(N-6)^{6}\left\langle p^{2}\right\rangle_{F}^{6}}
$$

and

$$
\left.\frac{1}{33} c_{2}\{8\}\right|_{p_{1}, \ldots, p_{8}} \approx \frac{24}{11} \frac{\left(p_{1} p_{2} p_{3} p_{4} p_{5} p_{6} p_{7} p_{8}\right)^{2}}{(N-8)^{8}\left\langle p^{2}\right\rangle_{F}^{8}} .
$$

\section{RESULTS}

In this section we plot $c_{2}\{k\}$ for different ranges of transverse momenta. We assume

$$
f(p) \propto \exp (-p / T),
$$

with $T=0.25 \mathrm{GeV}$. In this case $\langle p\rangle_{F}=2 T$ and $\left\langle p^{2}\right\rangle_{F}=6 T^{2}$.

In the left panel of Fig. 1 we present $\left(c_{2}\{2\}\right)^{1 / 2}$, $\left(c_{2}\{4\}\right)^{1 / 4},\left(c_{2}\{6\} / 4\right)^{1 / 6}$, and $\left(c_{2}\{8\} / 33\right)^{1 / 8}$ as a function of the number of particles, $N$. In this calculation all transverse momenta $p_{i}$ from Eqs. (14), (23), (26), and (27) are equal to $\left\langle p^{2}\right\rangle_{\Omega}^{1 / 2}$, where $\Omega$ is the transverse momentum range of $p>0.3$ $\mathrm{GeV}$, typically assumed in experimental measurements. The cumulants originating from transverse momentum conservation are basically inversely proportional to $N$, which reflects an intrinsic feature of TMC. They absolute magnitudes increase with the order of cumulant due to the larger coefficients present in higher-order cumulants, see Eqs. (14), (23), (26), and (27). It is interesting that their signs are consistent with the recent experimental measurement of $c_{2}\{k\}$, where for the lowest multiplicity events $c_{2}\{k\}>0$ [43].

In the right panel of Fig. 1 we present $\left(c_{2}\{2\}\right)^{1 / 2}$ as function of $p=p_{1}$ and $p_{2}=\left\langle p^{2}\right\rangle_{\Omega}^{1 / 2}$ for different values of $N=20,50$, and $100 .{ }^{6}$ As expected, the influence of TMC on $\left(c_{2}\{2\}\right)^{1 / 2}$ is

\footnotetext{
${ }^{5}$ In this case, the first nonvanishing terms in the expressions analogous to Eqs. (19) and (20) are given by $\Phi^{6} / 6$ ! and $\Phi^{8} / 8$ ! for six and eight particles, respectively.

${ }^{6}$ To be precise, we plot $c_{2}\{2\}\left(p, p_{2}\right) / \sqrt{c_{2}\{2\}\left(p_{2}, p_{2}\right)}$, which for Eq. (14) is equivalent to $\sqrt{c_{2}\{2\}(p, p)}$.
} 

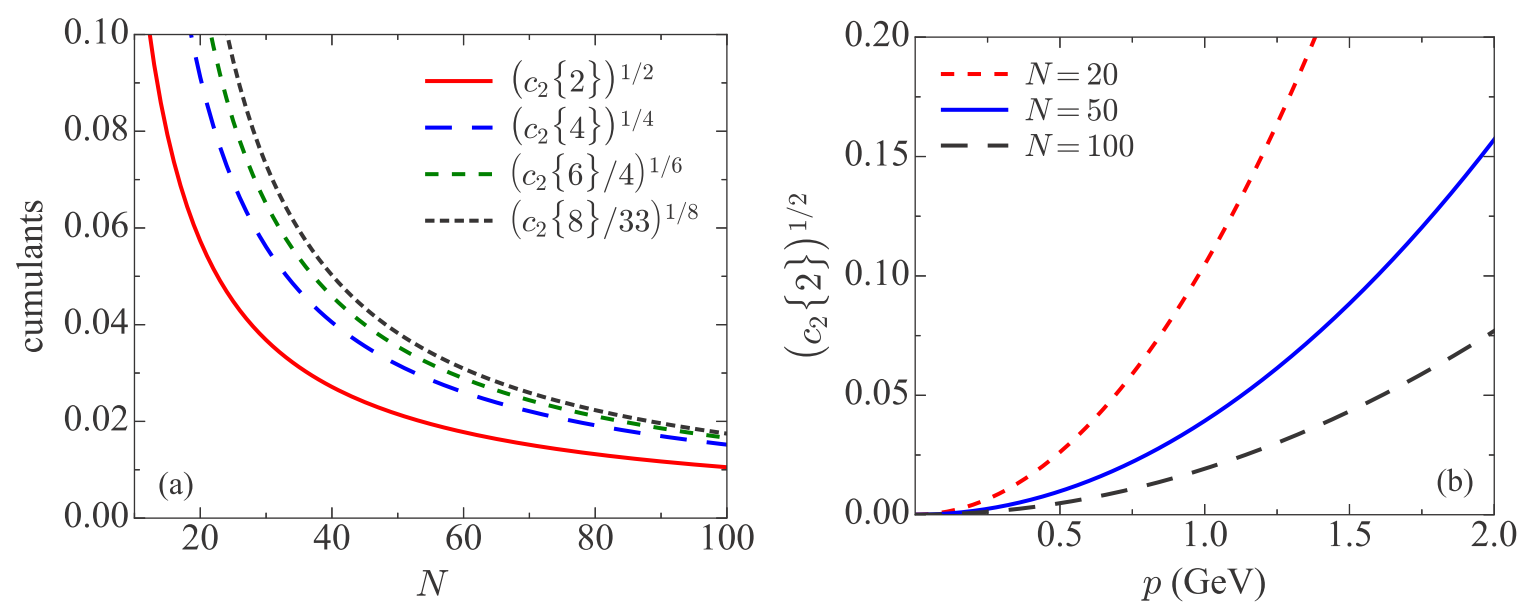

FIG. 1. (a) The multiparticle azimuthal cumulants $\left(c_{2}\{2\}\right)^{1 / 2},\left(c_{2}\{4\}\right)^{1 / 4},\left(c_{2}\{6\} / 4\right)^{1 / 6}$, and $\left(c_{2}\{8\} / 33\right)^{1 / 8}$ as a function of the number of particles, $N$. (b) $\left(c_{2}\{2\}\right)^{1 / 2}$ as function of transverse momentum, $p$, for different values of $N=20,50$, and 100 .

more significant for particles with higher momenta (parabolic dependence) and smaller number of particles $N$.

In addition, we calculated $c_{2}\{2\}$ using the Monte Carlo methods and obtained practically identical result to this shown in the left panel of Fig. 1. The purpose of this exercise was to verify that the central limit theorem, assumed in our analytical calculations, does not seriously effect the final results.

\section{CONCLUSIONS}

In this paper we calculated analytically the multiparticle elliptic flow cumulants originating from the conservation of transverse momentum. ${ }^{7}$ We demonstrated that $c_{2}\{k\}>0(k=$ $2,4,6,8)$ in qualitative agreement with the lowest multiplicity

\footnotetext{
${ }^{7}$ In our calculations we assumed that the conservation of transverse momentum is the only source of correlations between produced particles. It would be interesting to couple this mechanism with correlations originating from hydrodynamic flow. This should naturally explain the sign change of $c_{2}\{4\}$ and $c_{2}\{8\}$ as a function of the number of produced particles. This problem is currently under our investigation.
}

data in $p+p, p+A$, and $A+A$ collisions. As expected, the TMC contribution is found to be more significant for particles with higher transverse momenta and for systems with smaller number of particles (due to an intrinsic $1 / N$ effect). Depending on the transverse momenta, $\left(c_{2}\{k\}\right)^{1 / k}$ can reach substantial values, of the order of a few percentages, even for a relatively large number of particles. This effect should be taken into account when interpreting the long-range azimuthal correlations in small systems currently measured at RHIC and the LHC.

\section{ACKNOWLEDGMENTS}

We thank Piotr Bożek for useful comments. A.B. is partially supported by the Faculty of Physics and Applied Computer Science AGH UST statutory tasks within subsidy of Ministry of Science and Higher Education, and by the National Science Centre, Grant No. DEC-2014/15/B/ST2/00175. G.-L.M. is supported by the Major State Basic Research Development Program in China under Grant No. 2014CB845404, the National Natural Science Foundation of China under Grants No. 11522547, No. 11375251, and No. 11421505.
[1] J. C. Collins and M. J. Perry, Phys. Rev. Lett. 34, 1353 (1975).

[2] E. V. Shuryak, Phys. Rep. 61, 71 (1980).

[3] J. Adams et al. (STAR Collaboration), Nucl. Phys. A 757, 102 (2005).

[4] K. Adcox et al. (PHENIX Collaboration), Nucl. Phys. A 757, 184 (2005).

[5] K. Aamodt et al. (ALICE Collaboration), JINST 3, S08002 (2008).

[6] J. Y. Ollitrault, Phys. Rev. D 46, 229 (1992).

[7] E. V. Shuryak, Nucl. Phys. A 750, 64 (2005).

[8] H. Song, Y. Zhou, and K. Gajdosova, Nucl. Sci. Tech. 28, 99 (2017).

[9] N. Borghini, P. M. Dinh, and J. Y. Ollitrault, Phys. Rev. C 63, 054906 (2001).
[10] N. Borghini, P. M. Dinh, and J. Y. Ollitrault, Phys. Rev. C 64, 054901 (2001).

[11] C. Adler et al. (STAR Collaboration), Phys. Rev. C 66, 034904 (2002).

[12] C. Alt et al. (NA49 Collaboration), Phys. Rev. C 68, 034903 (2003)

[13] A. M. Poskanzer and S. A. Voloshin, Phys. Rev. C 58, 1671 (1998).

[14] A. Bilandzic, R. Snellings, and S. Voloshin, Phys. Rev. C 83, 044913 (2011).

[15] W. Florkowski, Phenomenology of Ultra-Relativistic Heavy-Ion Collisions (World Scientific, Singapore, 2010).

[16] D. Teaney, J. Lauret, and E. V. Shuryak, Phys. Rev. Lett. 86, 4783 (2001). 
[17] H. Song and U. W. Heinz, Phys. Rev. C 77, 064901 (2008).

[18] M. Luzum and P. Romatschke, Phys. Rev. C 78, 034915 (2008); 79, 039903(E) (2009).

[19] P. Bozek, Phys. Rev. C 81, 034909 (2010).

[20] B. Schenke, S. Jeon, and C. Gale, Phys. Rev. Lett. 106, 042301 (2011).

[21] V. Khachatryan et al. (CMS Collaboration), J. High Energy Phys. 1009 (2010) 091.

[22] B. Abelev et al. (ALICE Collaboration), Phys. Lett. B 719, 29 (2013).

[23] G. Aad et al. (ATLAS Collaboration), Phys. Rev. Lett. 110, 182302 (2013).

[24] S. Chatrchyan et al. (CMS Collaboration), Phys. Lett. B 724, 213 (2013).

[25] C. Loizides, Nucl. Phys. A 956, 200 (2016).

[26] A. Adare et al. (PHENIX Collaboration), Phys. Rev. Lett. 111, 212301 (2013).

[27] A. Adare et al. (PHENIX Collaboration), Phys. Rev. Lett. 115, 142301 (2015).

[28] D. McGlinchey (PHENIX Collaboration), Nucl. Phys. A 967, 19 (2017).

[29] L. D. Landau, Izv. Akad. Nauk SSSR, Fiz. Zemli 17, 51 (1953).

[30] S. Z. Belenkij and L. D. Landau, Nuovo Cimento Suppl. 3S10, 15 (1956); Usp. Fiz. Nauk 56, 309 (1955).

[31] P. Bozek, Phys. Rev. C 85, 014911 (2012).

[32] A. Bzdak, B. Schenke, P. Tribedy, and R. Venugopalan, Phys. Rev. C 87, 064906 (2013).

[33] E. Shuryak and I. Zahed, Phys. Rev. C 88, 044915 (2013).

[34] G. Y. Qin and B. Muller, Phys. Rev. C 89, 044902 (2014).

[35] P. Bozek and W. Broniowski, Phys. Rev. C 88, 014903 (2013).

[36] G. L. Ma and A. Bzdak, Phys. Lett. B 739, 209 (2014).

[37] A. Bzdak and G. L. Ma, Phys. Rev. Lett. 113, 252301 (2014).

[38] J. D. Orjuela Koop, A. Adare, D. McGlinchey, and J. L. Nagle, Phys. Rev. C 92, 054903 (2015).

[39] C. Shen, J. F. Paquet, G. S. Denicol, S. Jeon, and C. Gale, Phys. Rev. C 95, 014906 (2017).

[40] R. D. Weller and P. Romatschke, Phys. Lett. B 774, 351 (2017).

[41] K. Dusling, W. Li, and B. Schenke, Int. J. Mod. Phys. E 25, 1630002 (2016).

[42] M. Aaboud et al. (ATLAS Collaboration), Phys. Rev. C 96, 024908 (2017).
[43] M. Aaboud et al. (ATLAS Collaboration), Eur. Phys. J. C 77, 428 (2017).

[44] C. Aidala et al. (PHENIX Collaboration), arXiv:1707.06108 [nucl-ex].

[45] A. Dumitru, K. Dusling, F. Gelis, J. Jalilian-Marian, T. Lappi, and R. Venugopalan, Phys. Lett. B 697, 21 (2011).

[46] K. Dusling and R. Venugopalan, Phys. Rev. D 87, 094034 (2013).

[47] V. Skokov, Phys. Rev. D 91, 054014 (2015).

[48] B. Schenke, S. Schlichting, and R. Venugopalan, Phys. Lett. B 747, 76 (2015).

[49] S. Schlichting and P. Tribedy, Adv. High Energy Phys. (2016) 8460349.

[50] A. Kovner, M. Lublinsky, and V. Skokov, Phys. Rev. D 96, 016010 (2017).

[51] E. Iancu and A. H. Rezaeian, Phys. Rev. D 95, 094003 (2017).

[52] L. He, T. Edmonds, Z. W. Lin, F. Liu, D. Molnar, and F. Wang, Phys. Lett. B 753, 506 (2016).

[53] J. L. Nagle, R. Belmont, K. Hill, J. Orjuela Koop, D. V. Perepelitsa, P. Yin, Z. W. Lin, and D. McGlinchey, arXiv:1707.02307 [nucl-th].

[54] A. Ortiz, G. Bencedi, and H. Bello, J. Phys. G 44, 065001 (2017).

[55] T. A. Trainor, arXiv:1708.09412 [hep-ph].

[56] N. Borghini, P. M. Dinh, and J. Y. Ollitrault, Phys. Rev. C 62, 034902 (2000).

[57] N. Borghini, P. M. Dinh, J. Y. Ollitrault, A. M. Poskanzer, and S. A. Voloshin, Phys. Rev. C 66, 014901 (2002).

[58] Z. Chajecki and M. Lisa, Phys. Rev. C 78, 064903 (2008).

[59] Z. Chajecki and M. Lisa, Phys. Rev. C 79, 034908 (2009).

[60] S. Pratt, S. Schlichting, and S. Gavin, Phys. Rev. C 84, 024909 (2011).

[61] A. Bzdak, V. Koch, and J. Liao, Phys. Rev. C 83, 014905 (2011).

[62] G. Aad et al. (ATLAS Collaboration), Phys. Rev. C 86, 014907 (2012).

[63] J. Jia, M. Zhou, and A. Trzupek, Phys. Rev. C 96, 034906 (2017).

[64] M. Aaboud et al. (ATLAS Collaboration), arXiv:1708.03559 [hep-ex].

[65] V. V. Begun, M. Gazdzicki, and M. I. Gorenstein, Acta Phys. Pol. B 43, 1713 (2012). 